\title{
The Bennett Pinch for Non-Relativistic Electrons
}

\author{
J.E. Allen ${ }^{1,2,3}$ and L. Simons ${ }^{1} \dagger$ \\ ${ }^{1}$ Blackett Laboratory, Imperial College, London SW7 2AZ United Kingdom \\ ${ }^{2}$ OCIAM, Mathematical Institute, Oxford OX2 6GG, United Kingdom \\ ${ }^{3}$ University College, Oxford OX1 4BH, United Kingdom
}

(Received xx; revised $\mathrm{xx}$; accepted $\mathrm{xx}$ )

A theory of the Bennett pinch is presented for the case of non-relativistic electrons. In this way the basic physics of the phenomenon can be clearly demonstrated. The magnetic force acting on the electrons is transmitted to the positive ions via an electrostatic field.

\section{Introduction}

An early paper on the self-magnetic pinch effect was published by

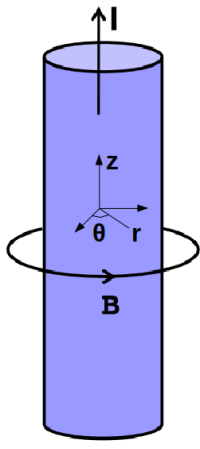
Bennett (1934) and discussed by Allen (2010) more recently at an E.P.S. conference. The well-known Bennett relation, to be described later, was originally obtained by considering two assemblies of particles, namely one of electrons and the other of positive ions. In the literature the subject is analysed using the theory of magnetohydrodynamics (MHD), but this approach can only give an incomplete description of the physics involved. Bittencourt (2004) considered the specific situation described by Bennett, but employed a balance between the (total) pressure gradient and the $\mathbf{j} \times \mathbf{B}$ force. In this way the existence of a radial electrostatic field was not revealed.

In this paper we shall consider a similar model, but for non-relativistic velocities. The self-magnetic pinch effect is illustrated in Fig.1. We

FIGURE 1. shall consider the electrons to have an average velocity $u$ in the $-z$ The pinch effect direction, together with a Maxwellian distribution of velocities described by a Temperature $T_{e}$. The positive ions will be considered to have a temperature $T_{i}$ and a negligible drift velocity, such a drift velocity could readily be included if necessary.

\section{Non-Relativistic Pinch Model}

Let us consider a coordinate system moving with the electron drift velocity. In this coordinate system an induced electric field of magnitude $u B$ exists in the radial direction, so that the total electric field in the radial direction is given by $E+u B$, where $E$ is the electric field in the laboratory frame of reference. The electron density is therefore given by

$$
n_{e}=n_{e 0} \exp \left(\frac{-e}{k_{B} T_{e}} \int_{0}^{r}(E+u B) d r\right)
$$

$\dagger$ Email address for correspondence: 1s5115@ic.ac.uk 
The electron density in thermal equilibrium depends upon the electric potential $V$ and is unaffected by the presence of a magnetic field. Individual particle orbits depend on the magnetic field, but macroscopic quantities are unaffected. This has been shown by Allen (1983) who pointed out that the Maxwell-Boltzmann distribution satisfies the Vlasov equation for a collision-free plasma in a magnetic field.

We can note that the electron density is unchanged when moving from one reference frame to another in a Galilean (or Newtonian) system. Eqn. (1) therefore can be taken to give the electron density in the laboratory frame of reference. In this reference frame the ion density is given by the Boltzmann relation

$$
n_{i}=n_{i 0} \exp \left(\frac{-e V}{k_{B} T_{i}}\right)
$$

The magnetic field is given by Ampères Law, which can be written as

$$
B=\frac{\mu_{0} e u}{r} \int_{0}^{r} n r d r
$$

We shall solve the problem using the concept of quasi-neutrality, i.e.

$$
n_{i}=n_{e}=n
$$

So that, using eqn. (1) and eqn. (2),

$$
V=\left(\frac{T_{i}}{T_{e}+T_{i}}\right) \int_{0}^{r} u B d r
$$

The electric field in the laboratory frame of reference is given by

$$
E=-\left(\frac{T_{i}}{T_{i}+T_{e}}\right) u B
$$

so that the force on the positive ions, per unit volume is $-\left(\frac{T_{i}}{T_{i}+T_{e}} n e u B\right)$, the negative sign indicating force towards the axis. The electric force acting on the electrons is $\left(\frac{T_{i}}{T_{i}+T_{e}}\right.$ neu $\left.B\right)$, i.e. an outward force. The magnetic force acting on the electrons is $-n e u B$ so that the net force on the electrons is $-\left(\frac{T_{e}}{T_{i}+T_{e}} n e u B\right)$. The total force on a unit volume of plasma is seen to be $-n e u B$ which can be identified as the $\mathbf{j} \times \mathbf{B}$ force employed in magnetohydrodynamics. The MHD model does not reveal the existence of the electric field which transmits part of the $\mathbf{j} \times \mathbf{B}$ force to the positive ions.

From eqn. (3) and (6) we can obtain the following

$$
E=-\frac{T_{i}}{T_{e}+T_{i}} \frac{1}{r} \int_{0}^{r} \mu_{0} e u^{2} n r d r
$$

From eqn. (2) we have

$$
n e E=k_{B} T_{i} \frac{d n}{d r}
$$

So that substitution in eqn. (7) gives

$$
\frac{d}{d r}\left(\frac{r}{n} \frac{d n}{d r}\right)+\frac{\mu_{0} e^{2} u^{2} n r}{k_{B}\left(T_{e}+T_{i}\right)}=0
$$

We shall look for a solution for the particle density $n$ (electron or ion) of the following form

$$
n=\frac{n_{0}}{\left(1+b n_{0} r^{2}\right)^{2}}
$$




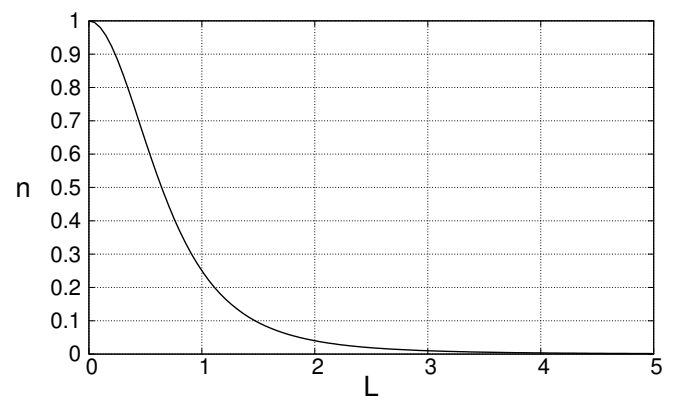

Figure 2. Profile of density with normalised radial distance $L^{*}=\left(b n_{0}\right)^{-0.5}$. The density is normalized by $n_{0}$.
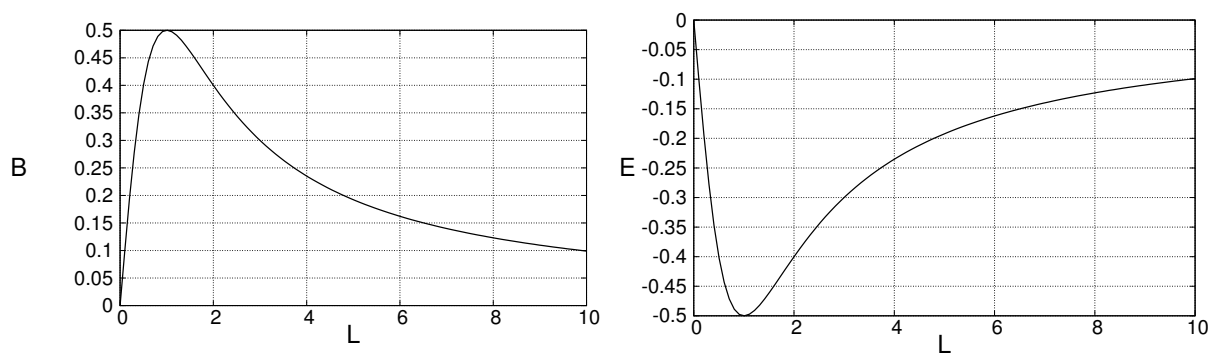

Figure 3. Profiles of electric and magnetic fields where the electric field is normalized by $E^{*}=\frac{T_{i}}{T_{i}+T_{e}} \frac{1}{2} \mu_{0} e n_{0} u^{2} L^{*}$ and the magnetic field by $B^{*}=\frac{1}{2} \mu_{0} e n_{0} u L^{*}$ with normalised radial distance $L^{*}=\left(b n_{0}\right)^{-0.5}$.

This function was originally found by Bennett (1934) to give the electron density in the fully relativistic case. It can readily be shown to be a solution of the present case of eqn. (9), where the quantity b, which has the dimensions of length, is given by

$$
b=\frac{\mu_{0} e^{2} u^{2}}{8 k_{B}\left(T_{e}+T_{i}\right)}
$$

The density profile is plotted in Fig. 2 .

Thonemann \& Cowhig (1950) found the solution for the particular case of $E=0$ (and hence $\left.T_{i}=0\right)$. In their experiments with a high current $(\sim 50 \mathrm{Amp})$ mercury arc, probe measurements indicated that the radial electric field was negligible.

The result of integrating eqn. (3) using eqn. (10) to express $n$ gives

$$
B=\frac{\mu_{0} e u}{r} \int_{0}^{r} \frac{n_{0} r}{\left(1+b n_{0} r^{2}\right)^{2}} d r=\frac{\mu_{0} e u n_{0} r}{2\left(1+b n_{0} r^{2}\right)}
$$

The radial variation of the magnetic field is shown in Fig. 3, together with the electric field as given by eqn. (6).

Using eqn. (12) the magnetic field at large radii is given by

$$
B=\frac{\mu_{0} e u}{2 b r}
$$


So that we can obtain the following expression for the current $I$, using Ampères Law,

$$
2 \pi r H=\frac{e u \pi}{b}=I
$$

but

$$
I=\int_{0}^{\infty} 2 \pi r n e u d r=N e u
$$

Where $N$ is the number of electrons (or ions) per unit length. Using eqns. (11), (14) and (15), we can write

$$
2 N k_{B}\left(T_{e}+T_{i}\right)=\frac{\mu_{0}}{4 \pi} I^{2}
$$

which is the well-known Bennett relation.

\section{Conclusions}

The non-relativistic model of the Bennett pinch clearly demonstrates the action of the $\mathbf{j} \times \mathbf{B}$ force in a collisionless plasma. The positive ions are contained by an electrostatic field (ignoring instabilities for the present discussion). This field results from an infinitesimal difference between the (high) electron and ion number densities. The concept of quasineutrality can be employed in this case. The inward electric force on the ions is $\left(\frac{T_{i}}{T_{i}+T_{e}} n e u B\right)$ and the inward net force on the electrons is $\left(\frac{T_{i}}{T_{i}+T_{e}} n e u B\right)$ per unit volume. The total inward force per unit volume of plasma is $\mathbf{j} \times \mathbf{B}$ as in a magnetohydrodynamics model, but the MHD model yields no information concerning the electrostatic field. A particular geometry has been considered in this paper, but the basic concept will apply to other geometries employed for the magnetic confinement of plasmas. It will also be of direct relevance to astrophysical plasmas; Alfvén (1970) has emphasized that a knowledge of the magnetic field configuration alone is insufficient to describe the "electromagnetic conditions".

\section{REFERENCES}

Alfvén, Hannes 1970 Plasma Physics, Space research and the origin of the solar system. In Nobel Lectures, Physics. (Elsevier, 1972).

Allen, J.E. 1983 A Paradox re-examined. European Journal of Physics 4 (3), 184-185.

Allen, J. E. 2010 The Bennett Pinch revisited. P4.181. 37th E.P.S. Conference on Plasma Physics, E.C.A., 34A.

Bennett, Willard H. 1934 Magnetically self-focussing streams. Physical Review 45 (12), 890-897.

Bittencourt, J. A. 2004 Fundamentals of Plasma Physics, 3rd edn. Springer US.

Thonemann, P. C. \& Cowhig, W. T. 1950 The Role of the Self Magnetic Field in High Current Gas Discharges. Proceedings of the Physical Society B 64, 345. 